\title{
NEGATIVE SCALAR CURVATURE METRICS ON NONCOMPACT MANIFOLDS
}

\author{
JOHN BLAND AND MORRIS KALKA
}

\begin{abstract}
In this paper we prove that every noncompact smooth manifold admits a complete metric of constant negative scalar curvature.
\end{abstract}

\section{INTRODUCTION}

If $M$ is a smooth compact manifold of dimension at least three, then according to [A], $M$ admits a metric of scalar curvature identically equal to -1 . It becomes a natural question whether any manifold of dimension at least three admits a complete metric of constant negative scalar curvature. This question was first posed by Kazdan in his January 1984 NSF Regional Conference lectures on Applications of Elliptic Partial Differential Equations to Differential Geometry.

In [BK] we announced a result in this direction and sketched the proof for $(n+1)$ dimensional manifolds when $n \geq 3$. The purpose of this paper is to supply the details missing in [BK], and to complete this result by showing the necessary modifications in dimension $3(n=2)$.

Theorem 1. Let $M$ be a noncompact manifold of dimension at least three. Then $M$ admits a complete metric of constant negative scalar curvature.

When $M$ has dimension 2 this result is well known. In particular, if $M$ is orientable one has the uniformization theorem for Riemann surfaces. Thus $M$ is covered by the hyperbolic disc and the covering transformations are hyperbolic isometries. If $M$ is nonorientable the result still follows from hyperbolic geometry but one needs an orientation reversing reflection. See e.g. Besse [B].

Since the statement of the theorem is simple and the main ideas in the proof can be easily explained, we include a rather lengthy introduction which gives a heuristic outline of the proof and which shows the ideas modulo some tedious but rather elementary calculus constructions. We hope that the effect of this is to make our paper easy to read. In broad outline, our approach is to cut the manifold up into a countable number of pieces each of which has finite

Received by the editors April 23, 1988 and, in revised form, March 15, 1989.

1980 Mathematics Subject Classification (1985 Revision). Primary 53C99.

Research of the first author partially supported by NSERC.

Research of the second author partially supported by Louisiana Board of Regents. 
topology. On each piece, a standard procedure produces a smooth metric with constant negative scalar curvature. In the two dimensional case we can insure that the boundary curves are simple closed geodesics of fixed length. This makes it easy to glue the pieces together along the geodesic curves. In the higher dimensional case, the glueing is more delicate and constitutes the main body of this paper.

We remark that $\mathrm{Z}$. Jin has given a counterexample to the Yamabe problem for noncompact manifolds [J]. Thus we cannot hope to prove our result by pointwise conformal transformation.

We use Morse theory to cut up the manifold by sublevel sets of the distance function to a point $p \in P$ as in [M]. As we have chosen a point $p$ with the property that the square of the distance function $r$ is a Morse function, it has at most countably many critical values which (after composition with a diffeomorphism of $\mathbf{R}$ ) we may assume to be a subset of the positive integers. We break up the manifold into pieces $C_{k}=\left\{x \mid k-\frac{1}{4}<r(x)<k+\frac{1}{4}\right\}$ and $A_{k}=\left\{x \mid k+\frac{1}{8}<r(x)<k+\frac{7}{8}\right\}$. Since the $A_{k}$ contain no critical points they are differentiable products $N_{k} \times I$ where $N_{k}=\left\{x \mid r(x)=k+\frac{1}{4}\right\}$. Since the $C_{k}$ contain all critical points, their topology can be quite complicated. We use the fact that compact manifolds admit metrics of constant negative scalar curvature in two essentially different ways. We will realize $C_{k}$ as an open subset of its double, $2 C_{k}$ and restrict a constant negative scalar curvature metric from $2 C_{k}$ to $C_{k} . A_{k}$, on the other hand, is differentiably a product $N_{k} \times I$. We use the constant negative scalar curvature metric $h$ on $N_{k}$ and form the product metric $d t^{2}+c h$ which has negative scalar curvature for each positive constant $c$. (Of course if $M$ is three dimensional, it could very well occur that $N_{k}$ is diffeomorphic to $S^{2}$. We show in Lemma 2.2 that in this case we can modify the Morse function so that $N_{k}$ has genus at least 2.) Then since the ends $C_{k} \cap A_{k}$ and $C_{k+1} \cap A_{k}$ are both differentiably $N_{k} \times I$, we will modify the metrics on them so that near the ends they are equal to $d t^{2}+c h$ for possibly different values of $c$.

On the open set $C_{k} \cap A_{k} \cong N_{k} \times I$ we have no a priori control on the metric obtained from the double of $C_{k}$; however, we can twist the end while leaving the boundary components fixed so that the metric is in the form $d t^{2}+g$, where the tensor $g$ has no $d t$ component (Proposition 1.1). To effect this change of coordinates we choose a level surface of $r$ in $C_{k}$ and let $t$ be the signed distance from the hypersurface. Then, at least for small values of $t$, the gradient flow of the function $t$ is simply the geodesic flow normal to the hypersurface. In general the flow lines will not agree with the flow lines induced by the gradient of $r$. However, since both sets of flow lines are transverse to the hypersurface, we can twist the end in such a way that the new flow lines for the function $t$ agree with the induced flow lines for the Morse function $r$. At the $k$ th stage we perform this operation on $C_{k+1} \cap A_{k}$ as well. 
We have now chosen our product coordinate $t$, so that there is a hypersurface $t=$ constant in $A_{k} \cap C_{k}$ and a neighborhood of this hypersurface on which the metric has the block form $d t^{2}+g$, where $g$ has no $d t$ component. We refer to this neighborhood as $U$. We alter the metric as follows: we consider metrics of the form

$$
d t^{2}+f(t)[\phi(t) g+(1-\phi(t)) h]
$$

where $\phi(t)$ is a cut-off function supported in $U$ and identically 1 in a neighborhood of the initial hypersurface. The function $f(t)$ is chosen to maintain scalar curvature bounded above by a negative constant (Proposition 1.2). That such an $f$ can be chosen is verified by writing down a second order ordinary differential inequality expressing the scalar curvature in terms of $f(t)$. We choose $f(t) \equiv 1$ near the initial hypersurface and sufficiently convex for positive values of $t$. Geometrically, the choice of $f$ corresponds to isometrically embedding the neighborhood $U$ where $f \equiv 1$ and then "flairing" the end so much that curvature in this direction dominates the curvature in the other directions.

When $t$ is sufficiently large, $\phi$ is 0 and the metric is of the form $d t^{2}+f(t) h$ (recall that $h$ is a metric of scalar curvature -1 on the cross section and is independent of $t$ ). We need to flatten out $f$ to a constant while keeping the sign of the scalar curvature; that is, we would like to obtain a metric of the form $d t^{2}+c_{k} h$ for some constant $c_{k}$. The special form of the metric allows us to do this (Proposition 1.4) by defining a piecewise smooth $C^{1}$ function with the desired properties and rounding off the corners. The smoothing can be done in such a way that the sign of the scalar curvature is maintained. An appropriate diffeomorphism of the $t$ variable must be chosen to allow enough time to flatten the metric.

These preliminaries accomplished we now discuss how to define the metric inductively. Choose a metric $h_{1}$ on $N_{1}$ and on $C_{1}$ choose a metric $g_{1}$ as above such that near the end $A_{1} \cap C_{1}$, the metric $g_{1}$ is of the form $d t^{2}+c_{1} h_{1}$. Scale the metric by a sufficiently small constant $\varepsilon_{1}$ so that the scalar curvature of $\varepsilon_{1} g_{1}$ is less than -1 and $\varepsilon_{1} c_{1}<1$. Assume now that we have constructed the metric out to $C_{k}$. Choose a metric $h_{k+1}$ on $N_{k+1}$ and construct a metric on $C_{k+1}$ as described above (that is, a product metric at the ends) and scale it so that the scalar curvature is bounded above by -1 . Then the metrics from $C_{k}$ and $C_{k+1}$ restrict to the ends of $A_{k}$ to metrics of the form $d t^{2}+c_{k} h_{k}$ and $d t^{2}+c_{k+1} h_{k}$, respectively, where $c_{k}<1, c_{k+1}<1$. Once again the scalar curvature of a metric of the form $d t^{2}+f(t) h_{k}$ can be computed in terms of $f(t), f^{\prime}(t)$ and $f^{\prime \prime}(t)$. A straightforward analysis (Theorem 1) shows that if the length of the interval $I$ in $A_{k}=N_{k} \times I$ is sufficiently long, then $f(t)$ can be chosen to interpolate between the two product product metrics $d t^{2}+c_{k} h_{k}$, $d t^{2}+c_{k+1} h_{k}$ while maintaining scalar curvature $<-\frac{1}{2}$. Completeness is assured by making $I$ have length at least 1 . 
The proof of the theorem for 3 manifolds requires extra care at various points in the argument. Aside from the topological constructions necessary in dimension 3, there are also technical problems in dimension three which arise from the second term in formula (1.10) for scalar curvature being positive instead of negative. We deal with these technicalities in $\S 2$.

\section{Proof of the theorem For $n \geq 3$}

According to [M] we can embed $M$ as a closed submanifold of $\mathbf{R}^{N}$ in such a fashion that for most points $p \in M, d_{\mathbf{R}^{N}}(p, \cdot)$ has only nondegenerate critical points. As a translation in $\mathbf{R}^{N}$ will not affect the property of nondegeneracy we can assume that $p$ is the origin and we define

$$
r: M \mapsto[0, \infty)
$$

by

$$
r(x)=d_{\mathbf{R}^{N}}(0, x) .
$$

By nondegeneracy, the critical points of $r$ are isolated and therefore discrete. Since there are at most countably many critical values of $r$, we may arrange that after a suitable diffeomorphism of $(0, \infty)$, the critical values are a subset of the positive integers. (We remark here that the case in which there are only a finite number of critical points is relatively easier; however, for expositional convenience, we treat it as a part of the general case.)

We define $C_{1}=\left\{x \mid r<\frac{5}{4}\right\}$ and for $k \geq 2, C_{k}=\left\{x \mid k-\frac{1}{4}<r(x)<k+\frac{1}{4}\right\}$. We further define $A_{k}=\left\{x \mid k+\frac{1}{8}<r(x)<k+\frac{7}{8}\right\}$. Since the critical values are at the positive integers, $A_{k}$ is diffeomorphic to $\left\{x \in M \mid r(x)=k+\frac{1}{4}\right\} \times I$. We denote by $N_{k}$ the codimension 1 submanifold

$$
N_{k}=\left\{x \in M \mid r(x)=k+\frac{1}{4}\right\} .
$$

Thus $A_{k} \cong N_{k} \times I$ and $M=\left(\bigcup_{k \geq 1} C_{k}\right) \cup\left(\bigcup_{k \geq 1} A_{k}\right)$. Let $2 C_{k}$ denote the double of $C_{k}$, a compact $n$-dimensional manifold. Since $n \geq 3,2 C_{k}$ admits a metric of scalar curvature identically -1 . Since $C_{k}$ is an open submanifold of $2 C_{k}$, the metric restricts to a constant scalar curvature metric on $C_{k}$. Since $A_{k} \cap C_{k} \neq \varnothing$ and $A_{k} \cap C_{k+1} \neq \varnothing$, the metrics on the various $C_{k}$ determine a metric of constant negative scalar curvature on the ends of $A_{k}$. The main point in what follows will be to extend the metrics to all of $A_{k}$ in such a manner that the scalar curvature is bounded above by a negative constant, independent of $k$. It will then follow from the work of Aviles and McOwen [AM] that the complete metric thus constructed can be conformally deformed to one of constant negative scalar curvature.

The first proposition will guarantee that we can choose a product structure on $A_{k}$ such that restriction of the metric coming from the $C_{k}$ 's to the ends of $A_{k}$ can be written in a block diagonal form. Once this product structure $A_{k} \cong N_{k} \times I$ is chosen accordingly, it will be considered fixed, and all remaining calculations will be essentially computations in one variable-the product coordinate. 
Proposition 1.1. There exists a product structure $A_{k} \cong N_{k} \times I$ such that in local product coordinates $\left(x^{\alpha}, t\right)$ for $A_{k} \cong N_{k} \times(-\varepsilon, 1+\varepsilon)$, the metrics from $C_{k}$, $C_{k+1}$ restrict to neighborhoods of $N_{k} \times\{0\}$ and $N_{k} \times\{1\}$ to metrics of the form $d t^{2}+G_{\alpha \beta}(x, t) d x^{\alpha} d x^{\beta}$.

Proof. The gradient vector field $\partial / \partial r$ induces a natural product structure $A_{k} \cong$ $N_{k} \times(-\varepsilon, T+\varepsilon)$ for some $\varepsilon, T>0$. The metrics from $C_{k}$ and $C_{k+1}$ restrict to $A_{k}$ to define a metric on a neighborhood of the ends of $A_{k}$, say, on $N_{k} \times(-\varepsilon, \varepsilon)$ and $N_{k} \times(T-\varepsilon, T+\varepsilon)$. Extend the metric smoothly to a metric on $N_{k} \times(-\varepsilon, T+$ $\varepsilon)$ and denote the associated inner product on vector fields by $\langle$,$\rangle . Define$ a new vector field $X$ on $N_{k} \times\{0\}$ and $N_{k} \times\{T\}$ by the conditions (1) $X$ is orthogonal to the hypersurfaces $N_{k} \times\{0\}$ and $N_{k} \times\{T\} ;(2)\left\langle X, \frac{\partial}{\partial r}\right\rangle>0$ and (3) $\langle X, X\rangle=1$. Extend $X$ to a neighborhood of the hypersurfaces by the condition that $\nabla_{X} X \equiv 0$, where $\nabla$ is the covariant derivative associated to the unique Riemannian connection; that is, $X$ generates flow along geodesics perpendicular to the respective hypersurfaces. The new vector field $X$ is defined now in neighborhoods of the hypersurfaces, say $N_{k} \times(-\delta, 2 \delta)$ and $N_{k} \times(T-$ $2 \delta, T+\delta)$, and we may assume further that $\left\langle X, \frac{\partial}{\partial r}\right\rangle>0$ where $X$ is defined (by possibly choosing a smaller $\delta>0$ ). Choose a smooth positive function $\phi$ with support contained in $N_{k} \times(\delta / 2, T-\delta / 2)$ and satisfying $\phi \equiv 1$ on $N_{k} \times[\delta, T-\delta]$. Define an extension of $X$, call it $Y$, by $Y=\phi\left(\frac{\partial}{\partial r}\right)+(1-\phi) X$. Then $Y$ is a smooth nonvanishing vector field on $N_{k} \times(-\delta, T+\delta)$. The flow along $Y$ defines a natural smooth map

$$
N_{k} \times[0, T+\delta) \rightarrow N_{k} \times[0, \infty) .
$$

Further, the image of the hypersurface $N_{k} \times\{T\}$ is a hypersurface in $N_{k} \times$ $(0, \infty)$ transverse to the vector field $Y$. Choose a smooth function $\psi>-1$ with support contained in $N_{k} \times(\delta, T-\delta)$ such that the flow along the renormalized vector field $Z=(1+\psi) Y$ defines a diffeomorphism from $N_{k} \times(0, T)$ onto $N_{k} \times(0,1)$. Notice that in a neighborhood of $N_{k} \times\{0\}$ and $N_{k} \times\{1\}$, the coordinate vector field $\frac{\partial}{\partial t}$ (where $t$ represents the product coordinate) agrees with the image of the vector field $Z$. Thus, by construction, the coordinates agree with Fermi coordinates in a neighborhood of the hypersurface $N_{k} \times\{0\}$ and $N_{k} \times\{1\}$, and in local coordinates $\left(x^{\alpha}\right)$ for $N_{k}$, the metric has the desired form $d t^{2}+G_{\alpha \beta}(x, t) d x^{\alpha} d x^{\beta}$. (This block diagonal form of the metric also follows immediately from conditions (1), (2) and (3) above, and the condition that $\nabla_{X} X \equiv 0$.)

Fix a metric on $N_{k}$ locally representable as $h_{\alpha \beta} d x^{\alpha} d x^{\beta}$, which has constant negative scalar curvature. When $\operatorname{dim} N_{k} \geq 3 \quad(n \geq 3)$, this is always possible by the result for compact manifolds [A]. When $\operatorname{dim}\left(N_{k}\right)=2$, this will be possible as long as the genus of the compact surface $N_{k}$ is at least 2 ; in $\S 2$, we will indicate how this can always be arranged through an appropriate choice for a Morse function. 
Let $\varphi(t)$ be a smooth function with support contained in $\left(\frac{\varepsilon}{2}, 1-\frac{\varepsilon}{2}\right)$ which is equal to 1 on $[\varepsilon, 1-\varepsilon]$. Define

$$
\hat{g}=d t^{2}+f(t)\left(\varphi(t) h_{\alpha \beta}(x)+(1-\varphi(t)) G_{\alpha \beta}(x, t)\right) d x^{\alpha} d x^{\beta} .
$$

Here $f(t)$ is a function yet to be determined. The next result gives us a choice for $f$.

Proposition 1.2. In equation (1.1) the function $f(t)$ can be chosen so that $R_{\hat{g}}$, the scalar curvature of $\hat{g}$, is $\leq-\frac{7}{8}$ on $\left(N_{k} \times(0,2 \varepsilon)\right) \cup\left(N_{k} \times(1-2 \varepsilon, 1)\right)$.

Proof. We denote coordinates on $N_{k} \times I$ for the sake of notational convenience by $\left(x^{0}, x^{\alpha}\right)$ with $x^{0}=t$ and Greek indices running from 1 to $n$.

Consider a metric of the form

$$
g=d t^{2}+g_{\alpha \beta}(x, t) d x^{\alpha} d x^{\beta} .
$$

A routine computation shows that

$$
\begin{aligned}
& \text { (a) } \Gamma_{00}^{0}=\Gamma_{00}^{\alpha}=\Gamma_{0 \alpha}^{0}=0, \\
& \text { (b) } \Gamma_{\alpha \beta}^{0}=-\frac{1}{2} \frac{\partial g_{\alpha \beta}}{\partial t}, \\
& \text { (c) } \Gamma_{0 \alpha}^{\beta}=\frac{1}{2} g^{\gamma \beta} \frac{\partial g_{\alpha \gamma}}{\partial t}, \\
& \text { (d) } \Gamma_{\alpha \beta}^{\gamma}=\widetilde{\Gamma}_{\alpha \beta}^{\gamma},
\end{aligned}
$$

where, as usual, $g^{\gamma \beta}$ denotes the inverse matrix to $g_{\alpha \beta}$, the summation convention is employed and $\widetilde{\Gamma}_{\alpha \beta}^{\gamma}$ denote the Christoffel symbols for the induced metric on the hypersurface $N_{k} \times\left\{t_{0}\right\}$. From this it is easy to see that the formula for the components of the Ricci tensor are

$$
\begin{aligned}
R_{\alpha \beta}= & \Gamma_{\alpha \beta}^{0} \Gamma_{0 \gamma}^{\gamma}+\Gamma_{\alpha \beta}^{\mu} \Gamma_{\mu \gamma}^{\gamma}+\frac{\partial \Gamma_{\alpha \beta}^{\gamma}}{\partial x^{\gamma}}+\frac{\partial \Gamma_{\alpha \beta}^{0}}{\partial t} \\
& -\Gamma_{\gamma \beta}^{0} \Gamma_{0 \alpha}^{\gamma}-\Gamma_{\gamma \beta}^{\mu} \Gamma_{\mu \alpha}^{\gamma}-\Gamma_{0 \beta}^{\mu} \Gamma_{\mu \alpha}^{0}-\frac{\partial \Gamma_{\gamma \beta}^{\gamma}}{\partial x^{\alpha}} .
\end{aligned}
$$

If we denote by $\widetilde{R}_{c \gamma \beta}$ the components of the Ricci tensor for the induced metric on $N_{k} \times\left\{t_{0}\right\}$, then

$$
R_{\alpha \beta}=\widetilde{R}_{\alpha \beta}-\frac{1}{2} \frac{\partial^{2} g_{\alpha \beta}}{\partial t^{2}}-\frac{1}{4} g^{\mu \gamma} \frac{\partial g_{\alpha \beta}}{\partial t} \frac{\partial g_{\mu \gamma}}{\partial t}+\frac{1}{2} g^{\mu \gamma} \frac{\partial g_{\gamma \beta}}{\partial t} \frac{\partial g_{\alpha \mu}}{\partial t}
$$

and

$$
R_{00}=-\frac{1}{2} \frac{\partial^{2}}{\partial t^{2}}\left(\log \operatorname{det} g_{\alpha \beta}\right)-\frac{1}{4} g^{\gamma \beta} g^{\mu \alpha} \frac{\partial g_{\alpha \gamma}}{\partial t} \frac{\partial g_{\beta \mu}}{\partial t}
$$


Taking the trace and denoting the scalar curvature of $N_{k} \times\left\{t_{0}\right\}$ by $\widetilde{R}$, we get

$$
\begin{aligned}
R= & \widetilde{R}-\frac{1}{4}\left(\frac{\partial}{\partial t}\left(\log \operatorname{det}\left(g_{\alpha \beta}\right)\right)\right)^{2}-\frac{\partial^{2}}{\partial t^{2}}\left(\log \operatorname{det}\left(g_{\alpha \beta}\right)\right) \\
& -\frac{1}{4} g^{\gamma \beta} g^{\mu \alpha} \frac{\partial g_{\alpha \gamma}}{\partial t} \frac{\partial g_{\beta \mu}}{\partial t} .
\end{aligned}
$$

Similarly if the metric $g$ is perturbed by

$$
\hat{g}=d t^{2}+f(t) g_{\alpha \beta}(x t) d x^{\alpha} d x^{\beta}
$$

then the scalar curvature is given by either of the two formulae below

$$
\begin{aligned}
\widehat{R}= & \frac{\widetilde{R}}{f}-\frac{1}{4}\left(\frac{\partial}{\partial t}\left(\log \operatorname{det}\left(g_{\alpha \beta}\right)\right)+n \frac{\partial}{\partial t}(\log f)\right)^{2} \\
& -\frac{\partial^{2}}{\partial t^{2}}\left(\log \operatorname{det}\left(g_{\alpha \beta}\right)+n \log f\right) \\
& -\frac{1}{4} g^{\gamma \beta}\left(\frac{\partial g_{\alpha \gamma}}{\partial t}+g_{\alpha \gamma} \frac{\partial}{\partial t}(\log f)\right)\left(\frac{\partial g_{\beta \mu}}{\partial t}+g_{\beta \mu} \frac{\partial}{\partial t}(\log f)\right) g^{\mu \alpha} \\
= & R+\left(\frac{1}{f}-1\right) \widetilde{R}-\frac{n^{2}}{4}\left(\frac{\partial}{\partial t}(\log f)\right)^{2}-n \frac{\partial^{2}}{\partial t^{2}}(\log f) \\
& -\left(\frac{n+1}{2}\right) \frac{\partial}{\partial t}\left(\log \operatorname{det}\left(g_{\alpha \beta}\right)\right) \frac{\partial}{\partial t}(\log f)-\frac{n}{4}\left(\frac{\partial}{\partial t}(\log f)\right)^{2} .
\end{aligned}
$$

We are now prepared to choose the $f(t)$ in the statement of the proposition. We first define $f$ to be identically 1 on $\left(0, \frac{\varepsilon}{2}\right)$. From the first equation in (1.7) it follows that if we maintain the inequality $f \geq 1$ on $(0,2 \varepsilon)$, then

$$
\widehat{R} \leq|\widetilde{R}|-\frac{\partial^{2}}{\partial t^{2}}\left(\log \operatorname{det}\left(g_{\alpha \beta}\right)\right)-n \frac{\partial^{2}}{\partial t^{2}}(\log f) .
$$

Recall from equation (1.1) that

$$
g_{\alpha \beta}=\varphi(t) h_{\alpha \beta}(x)+(1-\varphi(t)) G_{\alpha \beta}(x, t) .
$$

Define

$$
M\left(t_{0}\right)=\max _{\left\{t=t_{0}\right\}}\left(|\widetilde{R}|-\frac{\partial^{2}}{\partial t^{2}}\left(\log \operatorname{det} g_{\alpha \beta}(x, t)\right)\right) .
$$

It is trivially true, from (1.8), that $\widehat{R} \leq-1$ whenever $n(\log f)^{\prime \prime} \geq M(t)+1 .\left(^{\prime}\right.$ represents derivatives with respect to $t$.) Thus it would be sufficient to choose $F=\log f$ on $(0,2 \varepsilon)$ such that $F \equiv 0$ on $\left(0, \frac{\varepsilon}{2}\right)$ and $F^{\prime \prime} \geq \frac{1+M(t)}{n}$ on $\left(\frac{\varepsilon}{2}, 2 \varepsilon\right)$. Of course no such function could be smooth at $x=\frac{\varepsilon}{2}$. On the other hand, the two definitions can be patched together so that for any $\delta>0, \delta \geq F \geq 0$ and $\left|F^{\prime}\right| \leq \delta$ in an arbitrarily small neighborhood of $\left(\frac{\varepsilon}{2}-\alpha, \frac{\varepsilon}{2}+\alpha\right)$. Thus, for some $\alpha>0, \widehat{R} \leq-1$ on $\left(0, \frac{\varepsilon}{2}-\alpha\right) \cup\left(\frac{\varepsilon}{2}+\alpha, 2 \varepsilon\right)$, and it follows from the second equation of (1.7) that on $\left(\frac{\varepsilon}{2}-\alpha, \frac{\varepsilon}{2}+\alpha\right)$ 


$$
\widehat{R} \leq R-n F^{\prime \prime}+c(\delta, g)
$$

where the constant $c=0(\delta)$ as $\delta \downarrow 0$. Since near $t=\frac{\varepsilon}{2}, R$ is close to -1 and one can choose $F$ so that $F^{\prime \prime} \geq 0$, it becomes clear that $F$ can be chosen so that $\widehat{R} \leq-\frac{7}{8}$ on $(0,2 \varepsilon)$. This same argument works on $(1-2 \varepsilon, 1)$ completing the proof of Proposition 1.2.

Remark 1.3. We can guarantee that at $t=2 \varepsilon, f>1, f^{\prime}>0$ and $f^{\prime \prime} / f>$ $\left(f^{\prime} / f\right)^{2}$ so that $f^{\prime \prime} \geq 0$. This follows from the initial conditions $F=\log f=0$ and $F^{\prime}=0$, and the inequality $F^{\prime \prime}=f^{\prime \prime} / f-\left(f^{\prime} / f\right)^{2} \geq 0$.

We will now show how to extend $f(t)$ from being defined on $t \in\{(0,2 \varepsilon) \cup$ $(1-2 \varepsilon, 1)\}$ to $t \in I$. For convenience of notation one will take a change of $t$ variable (the new variable being denoted by $s$, and the constant $T$ to be chosen later) so that $A_{k} \cong N_{k} \times(-3 \varepsilon, T+3 \varepsilon)$ with $s=t-2 \varepsilon$ for $-\varepsilon<t<2 \varepsilon$ and $s=t+T-1+2 \varepsilon$ for $1-2 \varepsilon<t<1+\varepsilon$. In terms of the $s$-coordinates $\hat{g}=d s^{2}+f(s) h_{\alpha \beta}(x) d x^{\alpha} d x^{\beta}$ near $s=0$ and $s=T$ where $f(0)>1, f^{\prime}(0)>$ $0, f^{\prime \prime}(0) \geq 0$ and $\hat{g}$ has negative scalar curvature for $s<0$ and $s>T$.

Proposition 1.4. There is an extension of $f(s)$ to $\left\{0<s<s_{1}\right\} \cup\left\{T-s_{2}<s<\right.$ $T\}$ so that (i) there exist $c_{1}>0, c_{2}>0$, for which $f-c_{1}$ is zero to second order at $s_{1}$ and $f-c_{2}$ is zero to second order $T-s_{2}$; (ii) the scalar curvature of $\hat{g}$ is negative.

Proof. By equation (1.7), and using the fact that for $0<s<T$, the metric $g_{\alpha \beta}(x, t) d x^{\alpha} d x^{\beta}$ is the constant metric $h_{\alpha \beta}(x) d x^{\alpha} d x^{\beta}$ for which $\widetilde{R}=-1$

$$
\widehat{R}=\frac{-\left(1+n f^{\prime \prime}\right)}{f}-\frac{n(n-3)}{4}\left(\frac{f^{\prime}}{f}\right)^{2} .
$$

Since $n \geq 3$ the second term is nonpositive, and the inequality $\widehat{R}<1 / 2 f$ holds as long as $n f^{\prime \prime} \geq-1 / 2$. It is now clear that there exists a smooth extension of $f(s)$ from $s=0$ to $s=s_{1}$ which flattens $f$ out at $s=s_{1}$ (that is, $f=c_{1}$ vanishes to second order at $s=s_{1}$ for some constant $c_{1}$ ) as long as $s_{1}$ is sufficiently large (in particular, $s_{1} \geq 2 n f^{\prime}(0)$ ). A similar argument works for the second end. This fixes our choices for $s_{1}$ and $s_{2}$ and $T$ is chosen to be any number bigger than $s_{1}+s_{2}$.

After these preliminary observations we are ready to prove our main result.

Proof of Theorem 1 (Dimension $\geq 4$ ). Construct a metric $g_{k}$ on each $C_{k}$ as described above. In patching the metric on $C_{k}$ to one on $A_{k}$, a fixed product structure $A_{k} \cong N_{k} \times I$ is determined on the $A_{k}$, as well as a fixed metric $h_{k}$ on $N_{k}$ with constant negative scalar curvature. After choosing an appropriate diffeomorphism for the interval, say $A_{k} \cong N_{k} \times\left(-\varepsilon, T_{k}+\varepsilon\right)$, the metrics $g_{k}$ and $g_{k+1}$ restrict to $C_{k} \cap A_{k}$ and $C_{k+1} \cap A_{k}$ to a metric on the ends of $A_{k}$ which restricts to $N_{k} \times(0, \varepsilon)$ and $N_{k} \times(T-\varepsilon, T)$ as $d t^{2}+c_{k} h_{\alpha \beta} d x^{\alpha} d x^{\beta}$ and 
$d t^{2}+\tilde{c}_{k+1} h_{\alpha \beta} d x^{\alpha} d x^{\beta}$, respectively. Choose $\varepsilon_{k}$ so small that, 1) the scalar curvature on $\varepsilon_{k} g_{k}$ has a scalar curvature $\leq-1$ and, 2) both $\varepsilon_{k} c_{k}<1$ and $\varepsilon_{k} \tilde{c}_{k}<1$. (Notice that scaling $g_{k}$ by $\varepsilon_{k} g_{k}$ scales the scalar curvature by dividing by $\varepsilon_{k}$ ). (Scale $g_{1}$ on $C_{1}$ by $\varepsilon_{1} g_{1}$ such that $\varepsilon_{1} c_{1}<1$ and $\varepsilon_{1} g_{1}$ has scalar curvature $\leq-1$.) Finally, notice that on the ends of $N_{k} \times I$, the scaled metrics look like $\varepsilon_{k} d t^{2}+\varepsilon_{k} c_{k} h_{\alpha \beta} d x^{\alpha} d x^{\beta}$ and $\varepsilon_{k+1} d t^{2}+\varepsilon_{k+1} \tilde{c}_{k+1} h_{\alpha \beta} d x^{\alpha} d x^{\beta}$, but that after an appropriate diffeomorphism of the $t$ variable, it can be written as $d t^{2}+\left(\varepsilon_{k} c_{k}\right) h_{\alpha \beta} d x^{\alpha} d x^{\beta}$ and $d t^{2}+\left(\varepsilon_{k+1} \tilde{c}_{k+1}\right) h_{\alpha \beta} d x^{\alpha} d x^{\beta}$.

The completion of the proof of the theorem again splits into two cases, with the case $n \geq 3$ being the easier one. We will deal with this case here, again leaving the case $n=2$ to $\S 2$.

The relevant equation for the scalar curvature on $N_{k} \times(0, T)$ is again given by

$$
R=-\frac{\left(1+n f^{\prime \prime}\right)}{f}-\frac{n(n-3)}{4} \frac{f^{\prime} f^{\prime}}{f^{2}}
$$

and we wish to choose $f$ such that

$$
\left.f\right|_{(0, \varepsilon)} \equiv \varepsilon_{k} c_{k},\left.\quad f\right|_{(T-\varepsilon, T)} \equiv \varepsilon_{k+1} \tilde{c}_{k+1}
$$

and $R \leq-\frac{1}{2}$ on $(0, T)$. We will interpolate between $\varepsilon_{k} c_{k}$ and $\varepsilon_{k+1} \tilde{c}_{k+1}$ with a monotone function $f$, and since $0<\varepsilon_{k} c_{k}, \varepsilon_{k+1} \tilde{c}_{k+1}<1$, we may also require that $0<f<1$ on $(0, T)$. It is clear that it is sufficient to require that $-\left(1+n f^{\prime \prime}\right) / f \leq-1$ (since the other term has the correct sign when $\left.n \geq 3\right)$ and it is even sufficient to guarantee that $-\left(1+n f^{\prime \prime}\right) \leq-\frac{3}{4}$ (since $\left.0<f<1\right)$; that is, it is sufficient to interpolate between $\varepsilon_{k} c_{k}$ and $\varepsilon_{k+1} \tilde{c}_{k+1}$ with a monotone function $f$ which satisfies $n f^{\prime \prime} \geq-\frac{1}{4}$ or $f^{\prime \prime} \geq-\frac{1}{4 n}$. This is easy to do as long as $T>4 n+2$. In fact, one can easily check that a linear function of slope $\leq \frac{1}{4 n}$ in absolute value will interpolate between any two values between 0 and 1 on an interval of length less than $4 n$. At the end nearest the smallest of $\varepsilon_{k} c_{k}$ and $\varepsilon_{k+1} \tilde{c}_{k+1}$, this can be smoothly spliced to a constant function by a convex function $\left(f^{\prime \prime} \geq 0\right)$ which only helps the scalar curvature. At the end nearest the largest of $\varepsilon_{k} c_{k}$ and $\varepsilon_{k+1} \tilde{c}_{k+1}$, this can be smoothly spliced to a constant function by a concave function with $f^{\prime \prime} \geq-\frac{1}{4 n}$ over an interval of length 1 ( $f^{\prime}$ changes from 0 to $\frac{1}{4 n}$ over an interval of length 1 ).

The result is now easily established. On $C_{0}$, we choose our metric with scalar curvature $\leq-1$; this starts the process, and eliminates any problems with a finite portion of the topology. Similarly, on each $C_{k}, k \geq 1$, we choose our metrics as above. These metrics are spliced together over $N_{k} \times I$ in such a fashion that on any compact submanifold, the scalar curvature is less than $-\frac{1}{2}$. Since on $N_{k} \times(0, T)$, the metric is of the form $d t^{2}+f(t) h_{\alpha \beta} d x^{\alpha} d x^{\beta}$, and $T \geq 4 n+2>1$, the distance between the relatively compact sets $C_{k}$ is at least two, and the resulting metric is complete. 


\section{PROOF OF THE THEOREM FOR $n=3$}

As indicated in the introduction, the proof given in $\S 1$ has to be modified in 3 crucial places for 3 manifolds $(n=2)$. The first is a topological modification. Recall that the $A_{k}$ were topological products $N_{k} \times I$, and we used a metric on the cross section $N_{k}$ (=2 dimensional manifold) with constant negative scalar curvature. For $n=2$, this requires that the cross sections $N_{k}$ have genus $\geq 2$. This can always be attained through an appropriate choice of Morse function and will be the main content of Lemmas 2.1 and 2.2. The other critical places for $n \geq 3$ occur in Proposition 1.4 and the proof of the theorem. In both cases, equation (1.7) is used as

$$
\widehat{R}=-\frac{\left(1+n f^{\prime \prime}\right)}{f}-\frac{n(n-3)}{4} \frac{f^{\prime} f^{\prime}}{f^{2}} .
$$

When $n \geq 3$, the last term can be ignored. However, when $n=2$, the term is a positive contribution to the scalar curvature, and it must be dealt with more carefully. The modified Proposition 1.4 will be contained in Proposition 2.3, and the modified proof of the theorem will be presented as such.

We begin by considering the necessary topological modifications. The procedure which we employ (to add and subtract handles) is a standard topological technique. We include a description of it to make this paper self-contained for geometers.

Lemma 2.1. Let $D=\left\{\left(x^{1}, x^{2}, x^{3}\right) \in \mathbf{R}^{3} / 0<x^{1}, x^{2}, x^{3}<2\right\}$ be a threedimensional cube equipped with the Morse function $x^{3}$. Then there exists a Morse function $\varphi$ on $D$ with the following properties:

(i) $\left.\varphi\right|_{\partial D}=\left.x^{3}\right|_{\partial D}$.

(ii) The sets $\{\varphi=c\}$ for $c \in\left(0, \frac{1}{4}\right) \cup\left(\frac{5}{4}, 2\right)$ are topological discs.

(iii) The sets $\{\varphi=c\}$, for $c \in\left(\frac{1}{4}, \frac{5}{4}\right)$ are, topologically, discs with a handle attached.

Proof. We will describe the construction of $\varphi$ by a sequence of diagrams.

For small values of $c$, the sublevel sets $\{\varphi \leq c\}$ look like thin plates with flat tops. See illustration (1).

As the value of $c$ grows the surface of the plates grows small bumps in two disjoint places. See illustration (2).

As $c$ continues to increase, the bumps grow into horns sticking out from a flat plate. (Illustration (3).)

Thus far the level surface is still a topological disc. Now, however, we let the tips of the horns grow toward each other and touch. At the point where they touch, when $c=\frac{1}{4}, \varphi$ has a critical point and beyond this value the level sets are no longer simply connected. (Illustration (4).)

For $c \in\left(\frac{1}{4}, \frac{5}{4}\right)$, it now fills smoothly around the point where the horns first touched. As $c$ continues to grow, however, the handle grows fatter and fills in at the center. 
The surface which eventually fills in the center is a singular surface and thus this corresponds to a critical value for $\varphi$, which we may set to be $\varphi=\frac{5}{4}$. (Illustration (5).)

As $c$ grows past $\frac{5}{4}$, the level surface is simply connected and eventually becomes a flat plate again. Notice that the modification is carried on within a cube and that on the boundary of the cube the Morse function equals the Morse function $x^{3}$. (Illustration (6).)

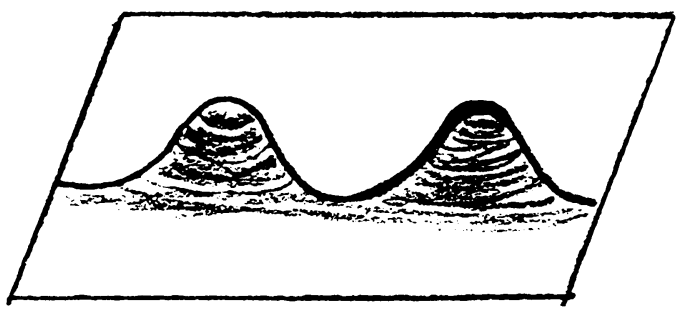

(1)

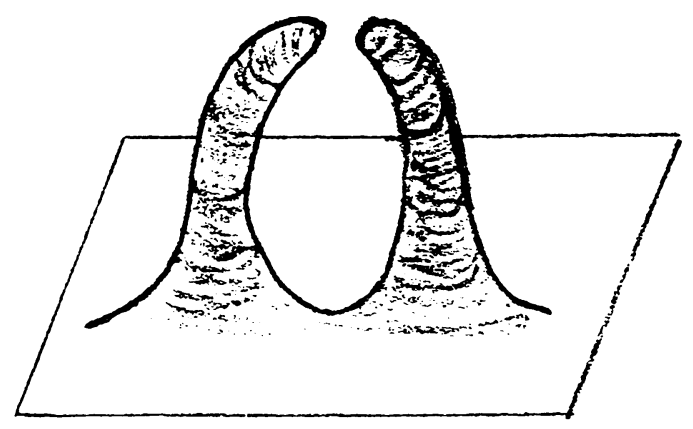

(2)

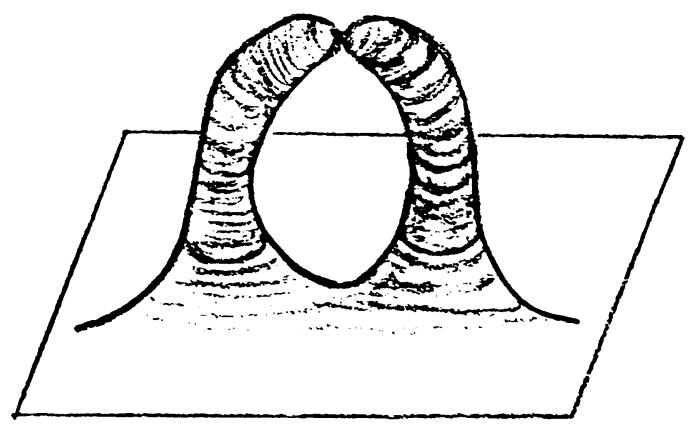

(3) 


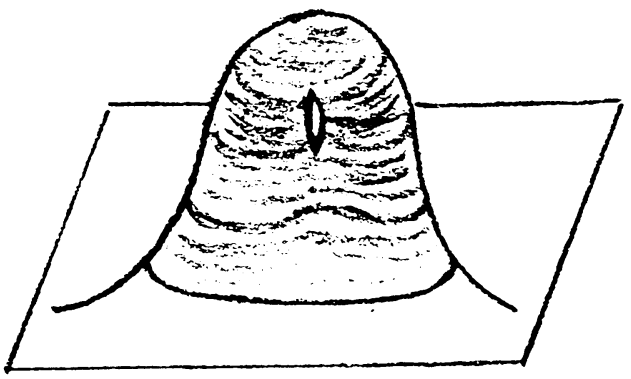

(4)

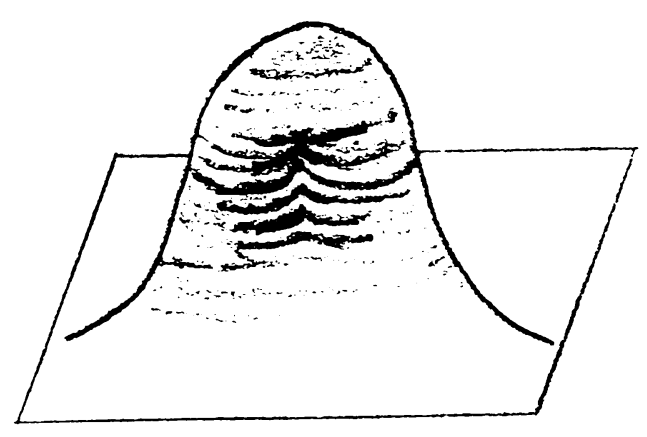

(5)

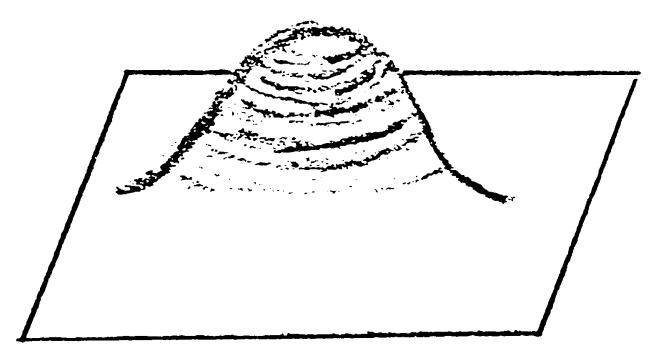

(6)

Lemma 2.2. For $n=2$, and with notation as in $\S 1$, we can modify the Morse function $r$ so that each level set $N_{k}$ is of genus at least 2 .

Since $r$ has only a finite number of critical points on each level set, choose two distinct regular points, say $p_{1, k}$ and $p_{2, k}$, for $r$ on each component of $\{r=k\}$. Let $U_{1, k}$ and $U_{2, k}$ be disjoint open neighborhoods of $p_{1, k}$ and $p_{2, k}$ on $\{r=k\}$ respectively with the property that all points in $U_{i, k}$ are regular points of $r$. We follow the gradient flow of $r$ through $U_{i, k}$. Since $r$ is a Morse function this intersects $\left\{r=k-\frac{1}{4}\right\}$ in disjoint open sets $U_{i, k-\frac{1}{4}}$. The gradient flow through $U_{i, k}$ intersects the critical level $\{r=k+1\}$ in sets $U_{i, k+1}$. Pick disjoint open subsets $V_{i, k+1} \subset U_{i, k+1}$ which have no critical points. Follow the forward flow of the sets $V_{i, k+1}$ to disjoint sets $V_{i, k+\frac{5}{4}} \subset\left\{r=k+\frac{5}{4}\right\}$ and the 
backward flow to disjoint open set $V_{i, k-\frac{1}{4}} \subset\left\{r=k-\frac{1}{4}\right\}$. By Morse Theory we have constructed two topological cubes to which we can apply Lemma 2.1.

The next proposition will be essentially the 3 dimensional analogue of Proposition 1.4 (for $n=2$ ). Recall that we have a metric on the ends of $N_{k} \times$ $(-3 \varepsilon, T+3 \varepsilon)$ of the form $\hat{g}=d s^{2}+f(s) h_{\alpha \beta} d x^{\alpha} d x^{\beta}$ where near $s=0$ and $s=T, \hat{g}$ has negative scalar curvature, and $f(0) \geq 1, f^{\prime}(0) \geq 0$, and $f^{\prime \prime}(0) \geq\left(f^{\prime}(0)\right)^{2} / f(0)$.

Proposition 2.3. There is an extension of $f(s)$ to $\left\{0<s<s_{1}\right\} \cup\left\{T-s_{2}<s<T\right\}$ so that (i) there exist constants $c_{1}$ and $c_{2}>0$ such that $\left(f-c_{1}\right)$ is zero to second order at $s_{1}$ and $\left(f-c_{2}\right)$ is zero to second order at $s=s_{2}$; ii) the scalar curvature of $\hat{g}$ is negative.

Proof. We will show how to flatten $f$ out only near the initial end $(f=0)$, the second end being an identical calculation. By (1.7) and using $n=2$, the scalar curvature is given by

$$
\begin{aligned}
\widehat{R} & =-\frac{\left(1+2 f^{\prime \prime}\right)}{f}+\frac{1}{2} \frac{f^{\prime} f^{\prime}}{f^{2}} \\
& =\frac{-1}{f}+\frac{1}{f}\left(-2 f^{\prime \prime}+\frac{1}{2} \frac{f^{\prime} f^{\prime}}{f}\right) \\
& =\frac{1}{f}\left(-1+\frac{1}{2} \frac{f^{\prime} f^{\prime}}{f}\right)-\frac{2 f^{\prime \prime}}{f} .
\end{aligned}
$$

$$
f^{\prime \prime}(0)>f^{\prime}(0) f^{\prime}(0) / f(0)>f^{\prime}(0) f^{\prime}(0) / 4 f(0) \text {. }
$$
The initial conditions imply that $f^{\prime \prime}(0)>f^{\prime}(0) f^{\prime}(0) / f(0)>f^{\prime}(0) f^{\prime}(0) / 4 f(0)$;
Thus, the second term in $\left(2.1_{b}\right)$ is negative, but we can smoothly reduce $f^{\prime \prime}$ until $f^{\prime \prime}=f^{\prime} f^{\prime} / 4 f$ at a point. Re-initialize this point to be $t=0$ and extend $f$ beyond $t=0$ by solving the initial value problem

$$
f^{\prime \prime} \equiv f^{\prime} f^{\prime} / 4 f
$$

subject to the new initial conditions $f(0)$ and $f^{\prime}(0)$. In particular, the general solution to the ordinary differential equation (2.2) is given by $f=(a+b t)^{\frac{4}{3}}$ where $a$ and $b$ are chosen to make $f$ continuously differentiable near $t=$ 0 . (This implies, in particular, that $a>0$ and $b>0$.) From $\left(2.1_{b}\right)$, it is clear that $\widehat{R}<-1 / f$ over this time interval. Further, as $t \rightarrow \infty, f^{\prime} f^{\prime} / f=$ $16 b^{2} / 9(a+b t)^{\frac{2}{3}} \rightarrow 0$. In particular, after a finite time, $f^{\prime} f^{\prime} / f<1$. At this point, $\left(2.1_{c}\right)$ shows that $\widehat{R}<(1 / f)(-1 / 2)-2 f^{\prime \prime} / f$. We can now reduce $f^{\prime \prime}$ to $-1 / 8$ in an arbitrarily small interval without changing the values of $f, f^{\prime}$ by some amount which is specified to maintain the inequality $f^{\prime} f^{\prime} / f<1$ and by $\left(2.1_{c}\right)$ the negativity of $\widehat{R}$. Then, since $f^{\prime \prime}<0$ and $f^{\prime}>0$, we have that $f^{\prime}$ is decreasing and $f$ is increasing, so $f^{\prime} f^{\prime} / f$ is decreasing and remains less than 1 . Equation $\left(2.1_{c}\right)$ shows that $\widehat{R}<\left(-1 / 2-2 f^{\prime \prime}\right) / f<-1 /(4 f)$ 
whenever $f^{\prime \prime}>-1 / 8$. It is now clear that $f$ can be flattened out (that is, $f^{\prime}$ can be reduced to be identically zero) in a finite time maintaining negative scalar curvature by maintaining $f^{\prime \prime}>-1 / 8$.

Proof of the theorem (for 3-manifolds). To complete the proof for three dimensional manifolds, it remains to show that given arbitrary constants $0<$ $c_{1}<c_{0}<1$, there exists a function $f$ defined on some finite time interval $(0, T)=\left(0, \frac{c_{0}-c_{1}}{c_{1}}+8 c_{1}\right)$ such that $f$ agrees to second order with the constant function $c_{0}$ at $t=0$ and the constant function $c_{1}$ at $t=T$ and such that

$$
\widehat{R}=\frac{1}{f}\left(-1-2 f^{\prime \prime}+\frac{1}{2} \frac{f^{\prime} f^{\prime}}{f}\right)
$$

satisfies $\widehat{R}<-\frac{1}{4}$. Then, the proof for the three dimensional case will follow exactly as for the higher dimensions.

Near $t=0, f^{\prime \prime}$ can be changed sufficiently quickly to $-\frac{1}{8}$ so that the values of $f, f^{\prime}$ are not significantly altered; by $(2.3), \widehat{R}$ is still less than $\left(-1+\frac{1}{2}\right) / f$. Next, solving the differential equation $f^{\prime \prime}=-\frac{1}{8}$ for $t \in\left(0,8 c_{1}\right)$ decreases $f^{\prime}$ from approximately 0 to $-c_{1}$. Now reduce $f^{\prime \prime}$ back to zero in an arbitrarily small interval without significantly affecting the value of $f^{\prime}$. Solve the linear differential equation $f^{\prime}=-c_{1}$ for $t \in\left(8 c_{1}, 8 c_{1}+\frac{c_{0}-c_{1}}{c_{1}}\right)$. Then $f$ is decreasing on $\left(0,8 c_{1}+\frac{\left(c_{0}-c_{1}\right)}{c_{1}}\right)$ and $f\left(8 c_{1}+\frac{\left(c_{0}-c_{1}\right)}{c_{1}}\right)<c_{1}$; thus there is a $t^{\prime} \in\left(8 c_{1}, 8 c_{1}+\right.$ $\left.\frac{\left(c_{1}-c_{0}\right)}{c_{1}}\right)$ for which $f\left(t^{\prime}\right)=c_{1}$. For $t \in\left(0, t^{\prime}\right), f^{\prime \prime} \geq-\frac{1}{8}, f>c_{1},\left|f^{\prime}\right| \leq c_{1}$ and by $(2.3)$

$$
f \widehat{R}<\left(-1+\frac{1}{4}+c_{1} / 2\right)<-\frac{1}{4} \text { or } \widehat{R}<-\frac{1}{4} c_{0} .
$$

Near $t=t^{\prime}$, by making $f^{\prime \prime}$ arbitrarily large positive for a sufficiently short time, we can reduce $f^{\prime}$ to zero rapidly and by $(2.3)$, only increase the negativity of $\widehat{R}$. Finally, extend $f$ as a constant for an interval of length 1 to insure completeness of the metric.

\section{REFERENCES}

[A] T. Aubin, Metriques riemanniennes et courbure, J. Differential Geom. 4 (1970), 383-424.

[AM] P. Aviles and R. McOwen, Conformal deformation to constant negative curvature on noncompact riemannian manifolds, J. Differential Geom. 27 (1988), 225-240.

[B] A. L. Besse, Einstein manifolds, Springer-Verlag, 1987.

[BK] J. Bland and M. Kalka, Complete metrics of negative scalar on noncompact manifolds, Contemp. Math., vol. 51, Amer. Math. Soc., Providence, R. I., 1986, pp. 31-35.

[J] Z. Jin, A counterexample to the Yamabe problem for complete noncompact manifolds, Partial Differential Equations, Lecture Notes in Math., vol. 1306, Springer-Verlag, 1988, pp. 93-101.

[M] J. Milnor, Morse theory, Ann. of Math. Studies, no. 51, Princeton Univ. Press, 1963.

Department of Mathematics, University of Toronto, Toronto, Ontario, Canada

Department of Mathematics, Tulane University, New Orleans, Louisiana 70118 